Cahiers de recherches médiévales

\title{
Le Lancelot-Guenièvre de Jean Cocteau
}

ou les Avatars du Mythe Amours, Sosies et Substitutions

\section{Robert Baudry}

\section{(2) OpenEdition}

Journals

Édition électronique

URL : https://journals.openedition.org/crm/2479

DOI : $10.4000 / \mathrm{crm} .2479$

ISSN : 1955-2424

Éditeur

Honoré Champion

Édition imprimée

Date de publication : 12 décembre 1996

Pagination : 37-49

ISSN : $1272-9752$

Référence électronique

Robert Baudry, «Le Lancelot-Guenièvre de Jean Cocteau », Cahiers de recherches médiévales [En ligne], 2 | 1996, mis en ligne le 04 février 2008, consulté le 15 décembre 2022. URL : http://

journals.openedition.org/crm/2479; DOI : https://doi.org/10.4000/crm.2479 


\title{
Le Lancelot-Guenièvre de Jean Cocteau
}

\author{
ou \\ les Avatars du Mythe \\ Amours, Sosies et Substitutions
}

Au firmament des couples d'amants célèbres, à côté de Tristan et d'Yseult, d'Abélard et Héloïse, de Roméo et Juliette, scintille aussi, de presque égale magnitude, cette étoile double de l'amour courtois: Lancelot et Guenièvre.

Faut-il rappeler ce qu'en rapporte la tradition légendaire? Lancelot du Lac s'est emparé du château de la Douloureuse Garde. Le roi Arthur y vient. A ses côtés, Lancelot aperçoit la reine. A l'instant, du premier regard, il tombe en extase d'amour, perdant conscience du temps et du lieu. Son ami Galehaut, fils de la Géante, sire des Iles Lointaines, s'entremettra pour que, dans le Pré aux arbrisseaux, Lancelot et Guenièvre se rencontrent et s'accordent. Voilà ce que raconte le Lancelot propre, le Lancelot en prose.

Rien d'étonnant à ce subit coup de foudre. Guenièvre' n'était-elle pas initialement une Fée, celle de la Souveraineté, qu'épousait le Roi ? Non que cette Fée royale ait laissé dans la légende réputation de dragon farouche, cuirassé de fidélité conjugale. Les Fées ses pareilles étaient, on le sait, accueillantes, accordant volontiers « l'amitié de leurs cuisses".

Guenièvre ne dément pas la coutume de ses sœeurs... Certaines triades galloises la rangeaient au nombre des femmes infidèles de l'île de Bretagne. Et Marie de France, qu'influençaient peut-être les clercs, incrimine sa légèreté. Il faut attendre le Chevalier à la charrette de Chrétien de Troyes pour que cette figure, un rien ambiguë, se nimbe d'amour courtois, de passion chevaleresque.

Pourtant, elle et Arthur s'étaient épousés par attirance réciproque, alors que, sur le conseil de Merlin, le jeune roi, encore contesté, séjournait à Carohaise comme simple chevalier au service du père de Guenièvre, le roi Léodagan de Carmélide. Les jeunes gens se virent. Ils se plurent. Et ils s'entr'aimaient fort ${ }^{2}$.

Si nulle aventure n'était prêtée à Guenièvre, Arthur, lui, n'était point tout novice en amour. Déjà, quand s'étaient rassemblés les barons pour élire un successeur à Uter Pendragon, ne s'était-il pas, à la faveur d'une absence du roi Lot d'Orcanie, glissé, écuyer facétieux, dans la couche de sa femme, belle et grasse, charmes dont il était épris. Celle-ci, à demiendormie, l'avait étreint, croyant embrasser son époux (ou le feignant...). Arthur ignorait que la reine fût sa demi-sceur. Inceste d'où naitra le sinistre Mordred qui, plus tard...

\footnotetext{
${ }^{1}$ Guenièvre : Gwenhwywar, Blanc Fantôme en gallois, que Geoffroy de Montmouth avait latinisée en Ganhumara, et dont les Anglais feront Jennifer.

${ }^{2}$ Cocteau avait pu en trouver l'écho dans l'adaptation de Jacques Boulenger, les Romans de la Table Ronde, L. I, p. 116, 136, 145, 197.

${ }^{3}$ Mordred, plus tard, devenu régent du royaume en l'absence d'Arthur, prétendra aux épousailles avec sa tante-marâtre Guenièvre - sorte d'Hippolyte inversé, ou de vizir Joseph poursuivant Putiphar. Et ces meurtres interdits du fils et du père effaceront de la terre les amours interdites du frère et de la scour, du neveu et de la tante. Tragédie familiale que raconte la Mort le Roi Artu.
} 
Voilà pour les amours d'Arthur. Celles de Guenièvre, qui ne les connait? Qui ne sait quels exploits Lancelot accomplit pour l'amour de sa Dame, si capricieuse, si jalouse fûtelle? Jalousie gratuite? - Oui! le plus souvent.

Pas toujours. Car, si jamais Lancelot ne lui fut infidèle en esprit, il lui advint de l'être de corps. C'est l'épisode d'Hélaine dans le Lancelot-Graal. Lancelot a visité le manoir du roi Pellés. Conseillé par sa maitresse Brisane, celui-ci administre à Lancelot un philtre. Abusé par lui, Lancelot prend sa fille Hélaine pour Guenièvre elle-même. Il la rejoint. Et de l'étreinte de cette unique nuit, naitra le futur chevalier du Graal, nommé Galaad, du vrai nom de son père. Aventure sans lendemain, dont Hélaine perdra, avec sa virginité, son office de porteuse du Graal.

On sait comment Lancelot défendra Guenièvre des prétentions d'une autre Guenièvre qui lui est faussement substituée 4 . On sait comme ensuite Lancelot, le Chevalier à la charrette de Chrétien, libèrera, nouvel Orphée, Guenièvre-Eurydice des mains de l'infernal Méléagant. On sait comme la vision du Graal lui demeurera interdite vu sa faute d'adultère. Qui ne sait tout cela?

- Un seul : Arthur, avec l'aveuglement habituel des maris trompés. Et comment en serat-il désabusé ? La fée Morgane, sœur d'Arthur, hait Guenièvre qui l'a séparée de son sigisbée Guiomar. Par traîtrise, Morgane réussit à emprisonner Lancelot. Deux hivers et un été, elle le retient dans son manoir de la Forêt Perdue. Pour tromper l'ennui de sa longue captivité, celui-ci imagine de retracer sur les murs de sa prison le récit de ses amours avec Guenièvre : c'est le Lancelot-propre.

Fatale imprudence! Car, lorsqu'à son tour, Arthur, égaré dans la Forêt Perdue, est hébergé chez sa sœur, celle-ci le loge à dessein dans cette «chambre aux images ». Au matin, ces fresques parlantes révèleront à Arthur les épisodes de sa conjugale infortune: c'est dans la Mort le roi Artu.

Bref rappel, qui n'était point inutile pour mieux mesurer à quel point Cocteau va réagencer toute cette tradition légendaire.

Tandis que le Roi Pêcheur (1940) de Gracq adaptera au théâtre le Parzival de Wolfram von Eschenbach, les Chevaliers de la Table Ronde (1937) de Cocteau apparaissent comme une variation du Lancelot-Graal. Car, dans cette pièce, alternent d'une part les péripéties qu'entraînent les amours de Lancelot et Guenièvre et, d'autre part, les aventures de la quête du Graal. Dans ce diptique, avant d'examiner ce qui conceme la quête du Graal, analysons d'abord l'autre volet : les amours de Lancelot et Guenièvre.

Dans l'ensemble, Cocteau reprend la donnée légendaire des relations adultères de Lancelot avec la reine, longtemps à l'insu de l'époux bafoué. Mais, dans ce triangle traditionnel, il modifie largement les rapports angulaires:

- Guenièvre a épousé Artus sans amour. Et Artus en fut dûment averti.

- Guenièvre n'est plus le premier et seul amour de Lancelot. Chez sa mère la Dame du Lac, il avait déjà épousé Mélusine et en avait eu un fils, Galaad.

- Lancelot et Guenièvre se rencontrent et s'aiment. Et tandis que d'Artus Guenièvre avait enfanté une fille, Blandine, aujourd'hui fiancée à Gauvain, de Lancelot elle avait également conçu un fils : Ségramor.

\footnotetext{
${ }^{4}$ Fils d'un faux mari substitué, Arthur ne méritait-il pas d'être abusé par une fausse Guenièvre?
} 
- Une fausse Genièvre tente, non plus de séduire Artus, mais de choquer Lancelot.

- Artus est éclairé sur son infortune, non point par Morgane et la chambre aux images, mais par Merlin et par un message expédié par une chauve-souris. Et Artus vérifie la trahison de la reine non par le témoignage des frères de Gauvain, mais par un rendez-vous où luimême se substitue à la reine dans les bras de Lancelot.

- Enfin, au lieu que Lancelot se fasse moine et Guenièvre nonne, ensemble ils rejoindront tous deux, et morts et immortels, le royaume invisible englouti sous les eaux, d'où les hèlent les Fées.

Telles sont les variations principales que Cocteau imprime au scénario canonique. Examinonsen de plus près le détail.

\section{Couple $n^{\circ} 1$ : Artus et Guenièvre}

"A tout seigneur, tout honneur»: d'abord, le couple royal. Cocteau ne peut, certes, esquiver - car c'est là une donnée fondamentale - le mariage entre Artus et « la Reine », - qu'il ne nomme jamais autrement; on se demande pourquoi... Mais, de ces noces, à quel point il modifie l'éclairage!

«Le roi savait en m'épousant, dit Guenièvre, que je ne l'aimais pas d'amour. Il a voulu m'épouser quand même et laissait mon couur libre.» (p.97)

Voilà ce qu'elle dit à Lancelot. Et voici ce qu'elle rappelle à Artus :

«Vous m'avez réduite à contracter un mariage sans amour. J'avais pour vous une amitié, un respect extrêmes. Je vous ai dit en toute franchise la nature de ma réserve et ma répugnance à me lier sans savoir ce qui pourrait advenir de mon coeur. Vous avez passé outre, vous m'avez répondu que mon couur restait libre, que l'amour naîtrait à la longue, que s'il ne naissait pas, vous vous contenteriez d'une amitié sainte, que j'étais trop jeune, que je ne comprenais pas. » (pp. 150-151).

Comme nous voilà loin des épousailles amoureuses d'Arthur et de Guenièvre selon la version consacrée du Lancelot-Graal! Mais pourquoi avoir ainsi transformé ce mariage d'inclination mutuelle en un ménage sans amour, avec autorisation maritale anticipée des fredaines possibles?

Réponse des optimistes: c'est pour mieux atténuer la faute d'adultère de Guenièvre une fois qu'elle aura rencontré Lancelot en en faisant son seul et unique amour.

Réponse des pessimistes: on peut y voir l'influence, anachronique, voire la satire de l'adultère mondain cher à notre temps, comme corollaire «normal » des mariages de convenance, princiers ou bourgeois, avec tolérance réciproque des " coups de canifs» que chacun apporterait au contrat. Et Artus, avouons-le, n'est guère moins ridicule en mari complaisant que - tranchons le mot, qui effraie les médiévistes - en mari cocu.

N'empêche qu'entretemps, cette « amitié sainte » d'Artus et Guenièvre avait, selon Cocteau, donné une fille, légitime. I la nomme «Blandine». Ce personnage est parfaitement inconnu - faut-il le préciser? - des « listings » de la tradition arthurienne. Son nom d'ailleurs n'a rien de celtique; il est tout latin. Serait-ce parce que ce fut une Blanchette Brunoy qui créa le rôle au théâtre ? 


\section{Robert BAUDRY}

Cocteau la présente comme fiancée à Gauvain, - son cousin puisque la pièce le confirme comme « neveu» d'Artus. (Il serait même son demi-frère si l'on en croyait certaines traditions qui donnent Gauvain comme produit des relations incestueuses d'Arthur avec sa (demi)-sœur).

Cette Blandine, Cocteau la relègue d'ailleurs dans un rôle effacé, plaintif, que troublent seulement les excentricités de son fiancé. En fait, il ne s'agit que d'un faux Gauvain, de qui un certain démon Ginifer a pris l'apparence, diablotin stupide, suppôt du néfaste Merlin.

Et voilà pour Guenièvre et Arthur. Face à eux, se situe un autre couple, tout aussi légitime : celui de Lancelot et de Mélusine.

\section{Couple $\mathrm{n}^{\circ} 2$ : Lancelot et Mélusine}

Lancelot! Qui est Lancelot? Les érudits ont beaucoup «phosphoré» autour de ce personnage assez énigmatique, sans que tout ce phosphore ait apporté - du moins aux profanes - une illumination définitive sur sa nature et ses origines. Peut-être même n'ont-ils fait qu'épaissir les mystères. Laissons donc cela à de plus savants pour en décider. Bornons-nous à ceci.

La vulgate légendaire, la seule qu'a pu connaitre Cocteau, fait de Lancelot le fils du roi Ban de Benoïc et d'une reine Hélène descendante du roi David. Adopté après le décès de son père par Viviane ou Niniane, la Dame du Lac, les romans bretons l'appelleront de son surnom de Lancelot du $\mathrm{Lac}^{6}$.

Cette ascendance humaine de Lancelot, Cocteau l'escamote pour en faire le fils, tout court, de la Dame du Lac. Du coup, c'est le «sang léger » des fées qui coule en ses veines, ce qui explique mieux son caractère « héroïque », au sens propre .

La version vulgate faisait de Guenièvre le premier et le seul amour de Lancelot ${ }^{8}$. Sur ce point aussi Cocteau s'affranchit de la tradition. C'est là, dans ce domaine magique du lac, que Lancelot aurait épousé en noces légitimes, l'une des fées de la suite de Viviane. Et quelle épouse lui prête-t-il ainsi ? Rien moins que Mélusine ${ }^{9}$, qu'il rameute donc d'un tout autre lignage, de la légende dynastique poitevine des Lusignan, bien étrangère au corpus arthurien des romans bretons. Et c'est de ces noces avec Mélusine qu'il fait naître, tout aussi légitimement, Galaad.

Pourquoi avoir été imaginer ce couple aberrant, ce mariage précoce? Pour plusieurs raisons.

\footnotetext{
${ }^{5}$ On imagine mal Cocteau se vouant à déchiffrer le Lanzelet en allemand d'Ulrich von Zatzikhoven

${ }^{6}$ Peut-être par jeu de mots sur un décalque français du gallois $L / w c h$ ayant donné approximativement Lac. Certains ont supposé que c'est suite à cette sorte d'étymologie populaire que Lancelot dit «du Lac » aurait été raccroché à la légende de la Dame du Lac.

${ }^{7}$ "Le sang léger coule dans tes veines... Fils des fées, nourri par les fées dans l'enchantement du Lac des fées, époux de Mélusine, tu regrettes les fées... ", lui dit Guenièvre, p. 98.

${ }^{8}$ Dans le Lanzelet, Lancelot parait au contraire comme un parfait polygame, épousant tour à tour trois jeunes filles dont il a préalablement oocis le père : un Cid doté d'une trinité de Chimènes...

${ }^{9}$ Il serait beau de suivre tous les avatars de Mélusine jusqu'à aujourd'hui. Nous l'avons tenté dans notre intervention "Les fées vivent toujours", lors du Colloque Fées, Dieux et Déesses au Moyen Âge, des 24 et 25 septembre 1993 à Lille III, dont les Actes ont été publiès dans Bien Dire et bien aprandre, Revue de médiévistique, Lille, 1994, $\mathrm{n}^{\circ} 12$.
} 
D'abord, pour Lancelot, en situant ces relations avant la rencontre avec Guenièvre, il lui évite le reproche d'infidélité pour son involontaire aventure d'une nuit avec la porteuse du Graal. En effet, on a vu comment, abusé par un philtre, il prendra pour Guenièvre Hélaine, la fille du Roi Pêcheur et comment cette "passade " sans lendemain engendrera Galaad" .

Ensuite, pour Galaad lui-même, voici qu'il n'est plus l'enfant bâtard d'une liaison irrégulière de Lancelot, ascendance trouble qui gêne quand même un peu quand il s'agit du "Très Pur".

En outre, le voici qui acquiert une ascendance féérique, et même des deux côtés, et par sa mère Mélusine et par son père Lancelot, fils tout court de la Dame du Lac. Filiation surnaturelle qui le prédispose peut-être mieux à sa vocation mystique de la quête du Graal.

Est-ce à dire que ces « améliorations » de l'intrigue légendaire n'entrainent aucun inconvénient? Si! Guenièvre n'est plus le seul et unique amour de Lancelot. Leurs relations deviennent doublement adultères, de part et d'autre. Et la quête et la conquête du Graal surnaturel par un Galaad, lui-même surnaturel, ôtent un peu au mérite héroïque de celui-ci.

On voit avec quelle prudence il convient de toucher aux données mythiques et comment la moindre "rectification » à la trame consacrée peut impliquer des répercussions imprévues. Le mythe se défend: nous l'avons dit ailleurs.

Puis, Lancelot se sépare, sans grand déchirement semble-t-il ${ }^{11}$, de Mélusine. Il quitte le lac des fées. C'est alors que va lui apparaître sa reine, Guenièvre, l'amour de sa vie. Et c'est :

\section{Le couple $n^{\circ} 3$ : Lancelot et Guenièvre}

Aux yeux de Guenièvre, le Lancelot de Cocteau apparait avec toute la séduction du «légionnaire»:

«Lancelot vint. Il arrivait du Lac. II était environné d'audace, de surnaturel et d'une sorte de phosphorescence. II était jeune, beau, brave, irésistible... Vous d'abord [Artus], nul ne résistait à son prestige » (p. 151)

Guenièvre n'y résistera pas... Et de cet éblouissement réciproque naquit le couple d'amants célèbres. Liaison que vient donc sanctifier l'amour selon un leit-motiv consacré depuis le romantisme, et dont Guenièvre ose même rendre la Providence complice : « Notre amour est de Dieu, j'en suis sûre », prétend-elle (p.95) ; et, plus loin, elle insiste et persiste:

«L'amour que je te porte, que je t'ai porté par grande merveille dès notre première rencontre est un amour de Dieu... S'il te semble que cet amour vienne du diable, tu n'en goûteras plus la douceur. " (p. 97)

Autre manière, certes, d'atténuer la faute adultère. Au moment où commence la pièce, voilà dix-huit ans que dure et se cache cet amour « fidèle ». Et voilà deux ans que Lancelot, bridé par ce mors affectif, a renoncé à courir les aventures, quitte à ronger en cachette son frein chevaleresque.

\footnotetext{
${ }^{10}$ Mais il prête à Galaad les enfances de Perceval : «Un soir, dans une forêt, j'ai vu passer vos chevaliers, et je les ai pris pour des anges. J'ai voulu les suivre. J'ai longtemps erré sans découvrir votre royaume. », p. 104.

${ }^{11}$ \& J'ai quitté Mélusine en pleine clairvoyance. En rompant le pacte, j'étais prévenu que c'était définitif. Il m'arrive de penser à mon fils, je l'avoue », p. 98.
} 


\section{Robert BAUDRY}

Rencontre, coup de foudre immédiat, longue liaison clandestine...: Cocteau n'innove guère sur ce point.

Là où il bouscule les données légendaires, c'est quand, à ce couple d'amants, il prête un fruit de leurs relations amoureuses : un fils. Et quel fils! - Ségramor! "Ségramor est le fils de notre faute ", dit Lancelot (p. 95).

Voilà qui est fort étrange. Car ce personnage, le cycle arthurien le connaît sous le nom tantôt de Sagremor ou Saigremor, tantôt de Ségramor ${ }^{12}$. C'est cette forme, héritée notamment de Wolfram von Eschenbach, qu'adopte Jean Cocteau.

Rejeton étrange, oui! Et même étranger. Car la tradition le donne communément comme " neveu de l'empereur de Constantinople ${ }^{13}$. Cocteau le soustrait à cette dynastie byzantine pour l'introduire - par fraude - dans le lignage arthurien comme fils putatif d'Artus ${ }^{14}$. Il devient ainsi, par Guenièvre, demi-frère de " Blandine » et, par Lancelot, demi-frère de Galaad.

En dépit de ses accointances impériales, la tradition médiévale ne lui réserve que des rôles assez secondaires ${ }^{15}$. Erec et Enide le cite dans son palmarès des chevaliers de la Table Ronde. Mais il n'y recueille qu'un maigre accessit au vingt-septième rang sur trente-trois. Chez Cocteau, sa nouvelle filiation, censément arthurienne, le hisse du vingt-septième au troisième rang dans le protocole de l'ordre de la Table Ronde, tout de suite après Lancelot, l'amant, et Gauvain, le neveu ; mais avant Merlin et « Beaudémagu ». - Sang breton oblige!

Ce Sagremor, la tradition l'affuble du sumom « le Desrøez" c'est-à-dire le Déréglé, le Détraqué, épithète de nature qui lui colle au corps. On sait comment, le premier, il vient déranger le Perceval de Chrétien en extase devant le sang des oies sur la neige. Et Wolfram renchérira sur la sottise de cet énergumène ${ }^{16}$ (il va jusqu'à découvrir au petit matin le roi et la reine couchés ensemble dans leur lit!)

\footnotetext{
${ }^{12}$ Sagremor (maudit More ?) apparaît chez Chrétien de Troyes dès Cligès et Erec et Enide. Perceval ou le Conte du Graal l'appelle Sagremor selon l'édition Hilka, Saigremor(s) selon l'édition Roach. Wolfram von Eschenbach adopte la forme Segramor, peut-être par une de ces variations étymologiques favorites : amour secret, plutôt que More maudit...

${ }^{13}$ La Continuation de Gerbert de Montreuil lui accorde aussi deux frères évêques.

${ }^{14}$ Pourquoi Cocteau a-t-il imaginé de transformer ce transfuge byzantin en héritier d'Artus ? Peut-être en greffant une erreur sur une faute de lecture de Wolfram... Chrétien l'avait qualifié de " petit jeune» ( « jeune poys ", v. 3845). De ce «jeune poys», Wolfram avait fait un «jeune roi ». Et, de ce «jeune roi $"$, Cocteau aurait fait un « jeune $d u$ roi »...-A quoi tiennent les titres!

${ }^{15}$ Cligès le vainc en tournoi (v. 46660). L'Erec d'Erec et Enide le secourt à Tenebroc (v. 1701, 2175, 2182, 2194). Il y est réputé « uns chevaliers de molt grant pris » (v. 2183). Mais l'Erec en prose l'ignore, tout comme le Chevalier a la Charrette. Dans la Première Continuation de Perceval, il joue sa partie dans divers combats avec des succès variables ; le plus souvent, il fait nombre dans la liste des chevaliers (v. 5449, 8379, 8548, 8865, 12933). Les autres Continuations le citent assez fréquemment, notamment celle de Gerbert de Montreuil (fol. 1000). Bref, ce n'est pas une lumière, un phare, mais une lampe d'appoint. On le retrouve dans Perlesvaus, le Chevalier as deus Espees, la Mort le roi Artu, le Didot-Perceval et le Merlin-Huth. mais il est inconnu du Roman de l'Estoire dou Graal et de la Queste del Saint Graal. Il réapparait dans maints romans chevalenesques: le Bel Inconnu, Durmart, Escanor, Fergus, Floriant, Hunbaut, Rigomer (fol. 178), le Tristan en prose, Hertz et Clanis et Laris. Il opèrera une rentree en force dans le Parzival und Titurel de Wolfram von Eschenbach.

${ }^{16}$ Wolfram en trace un portrait pittoresque : « Ségramor ne rêvait que bataille. Il ne marchait pas ; il courait par bonds. Dès qu'il trouvait une occasion de se battre, il fallait l'attacher." Arthur doit tanoer ce « jeune jouvenceau imberbe » : qu'il cesse de se conduire à l'étourdie comme un "héros écervelé "
} 
Ce caractère de Matamore impétueux est fort édulcoré chez Cocteau. Sans doute fut-il présomptueux en tentant témérairement l'épreuve du Siège Périlleux. Ce qui lui vaut de garder sur sa poitrine le stigmate du Coup Douloureux qui le châtia de son outrecuidance ${ }^{17}$.

Mais, depuis cette péripétie advenue avant le lever du rideau, notre Ségramor semble bien assagi. Il est même décrit comme un poète, bénéficiant de cette révélation d'ordre magique qui consiste à comprendre le langage des oiseaux.

\section{Couple $n^{\circ} 4$ : Lancelot-Ginifer (alias la fausse Reine)}

Ces amours illuminées de Guenièvre et Lancelot ne vont point toujours sans nuage. Ici, chez Cocteau, ce n'est plus une Hélaine porteuse du Graal, ni une Passerose demoiselle d'Escalot qui éveillent la jalousie de Guenièvre. C'est l'interférence d'une fausse Guenièvre qui vient les traverser.

En fait, cette "femme" n'est autre que le jeune démon Ginifer, suppôt de Merlin, qui a pris l'apparence de la Reine ${ }^{18}$. C'est Merlin qui a machiné ce double de Guenièvre. Par là, il escompte troubler l'amour entre les amants et égarer la quête du Graal qui démasquerait ses manigances.

Comment a pu germer dans l'esprit de Cocteau l'idée de pareille substitution? Certes, de Berte aus grans piés d'Adenet le Roi (vers 1280) au Roman de Macaire (XIII siècle), les récits médiévaux ne sont point avares de fausses reines ni de reines substituées.

Point n'est besoin toutefois de chercher si loin. Le cycle arthurien nous offre déjà une "Guenièvre-bis ». Le Lancelot propre évoque une demi-sœur de Guenièvre, nommée également Guenièvre, fille également du roi Léodagan, mais bâtarde qu'il aurait eue d'une liaison avec la femme de son sénéchal.

Parfaite sosie de la reine, comment cette «copie conforme " ne songerait-elle pas à supplanter l'original sur le trône et dans le lit d'Arthur? Après une première tentative déjouée par Merlin, elle parvient tout un temps à se faire passer pour la vraie reine et à abuser Arthur. Lancelot seul, sacrifiant héroïquement sa chance auprès d'une Guenièvre répudiée, réussira à rétablir sa Dame dans ses droits usurpés, à la remettre dans les bras de son officiel époux.

(traduction, p. 249), un «matamore outrecuidant» (p. 250). Il se défend d'être fou $a$ lier, « homme qu'on doive attacher avec des cordes ", (cf. 421,20 et 285,1 ).

Les Romans de la Table Ronde proposent une autre étymologie pour ce surnom embarrassant : " La reine l'avait surnommé le desréé parce qu'il était toujours le premier à se détacher, à quitter les rangs et à courir à l'ennemi. A lui toujours la première lance ; à lui de combattre à l'extréme pointe, en enfant perdu. " Et ils ajoutent ce détail pittoresque : « Le desréé avait une maladie : quand il s'était échauffé à combattre, il lui fallait manger. Sinon, en se refroidissant, il enrageait de faim et s'affaiblissait à en mourir. C'est pourquoi Keu l'avait sumommé Mort-de-Jeûne."

${ }^{17} \mathrm{Car}$ le Coup douloureux, Cocteau l'associe à l'épreuve du Siège périlleux. Coup qui agit de lui-même sans que nul ne le porte. Par contre, ce Coup douloureux est dissocié de la dévastation du royaume : le "Gaste Pays » est réputé dériver de la malédiction du Graal. En fait il résulte des manigances néfastes de Merlin. - Réagencement des thèmes fondamentaux du mythe, qui confirme à nouveau que ce n'est point tant le rapport établi entre eux qui comple que leur présence, d'une façon ou d'une autre. Cf. note 20.

${ }^{18}$ Ce nom de Ginifer parait d'ailleurs (tout comme le « Jennifer » moderne) un doublet linguistique de Guenièvre. 


\section{Robert BAUDRY}

Faut-il chercher là l'embryon de l'innovation de Cocteau? Cette intervention d'une fausse Reine, Cocteau avait pu la trouver dans quelque adaptation des Romans de la Table ronde, telle celle de Jacques Boulenger. Quoi qu'il en soit, dans sa pièce, la fausse Reine acquiert une nature et joue un rôle bien différents.

D'abord, ce n'est plus un Merlin tutélaire, protecteur du royaume, qui tente de conjurer l'imposture. C'est lui au contraire, génie malfaisant, dévorateur du domaine, qui se fait l'instigateur de cet équivoque dédoublement.

Ensuite, ce n'est plus le ménage d'Arthur que cette substitution prétend perturber, mais bien au contraire les amours de Lancelot. Heureusement le diablotin transformé en reine est si stupide que Lancelot aura bien du mal à reconnaître son amie dans cette virago excentrique (esscentrique, dirait-elle...).

Enfin, ce n'est plus une femme bien réelle, bien humaine, qui veut supplanter la reine, mais un diablotin - un diablotin mâle - qui prétend à l'amour du viril Lancelot. Couple certes bizarre! Ne s'expliquerait-il pas par un écho voilé, peut-être insconscient, de certaines tendances bien connues de Cocteau ? Ce ne serait pas la première fois qu' un auteur exprimerait, par le détour du mythe, un symbole de ses fantasmes profonds.

\section{Couple ${ }^{\circ} 5$ : Artus et Lancelot!}

Mari trompé, Artus ignorera longtemps la trahison de son épouse - dix-huit ans dans la pièce de Cocteau!

On a vu comment, dans la vulgate légendaire, Arthur découvre enfin les récréations extra-conjugales de sa royale Guenièvre. Egaré dans la Forêt Perdue, il trouve asile dans le château de sa (demi-)sceur Morgain. Celle-ci, qui déteste Guenièvre, le loge à dessein dans la « chambre aux images », celle où naguère Lancelot qu'elle y retenait prisonnier, avait, pour tromper l'ennui de sa captivité, retracé sur les murs l'histoire sans voile de ses amours avec la reine (Lancelot en prose, cf. Jean Marx, la Légende arthurienne et le Graal, p. 355). Au matin, Arthur découvrira ces éclairantes peintures (la Mort le roi Artus, cf. ibid, p. 370).

Tout autre est la manière dont Cocteau s'y prend pour informer Artus de son infortune. Cette fois, c'est un autre magicien, devenu néfaste, à savoir Merlin, qui, du Château noir qu'il a hérité de son collègue Clingsor, expédie à Arthur par une chauve-souris - variante nocturne du « corbeau »... - un message qui lui révèle les frasques de sa femme (p. 149).

Le roi l'interroge: - est-elle coupable d'une telle forfaiture ? Elle tente de s'en tirer par une série d'astuces - que certains diront bien «féminines». Après avoir rappelé à quel pacte de noces sans amour et de cœur laissé libre Arthur avait initialement souscrit, voyez par quelles roueries, en jouant sur les mots, Guenièvre élude toute réponse nette, réussit à ne jamais répondre aux questions, même les plus précises, sinon par d'autres questions, à la manière (dit-on) des rabbins ou des jésuites...

«I était jeune, beau, brave, irrésistible...Vous d'abord, nul ne résisterait à son prestige. Et vous l'aimiez et nous l'aimions et je l'aime, et quels griefs me faites-vous d'aimer ce que vous aimez, que chacun aime, d'admirer ce que tous admirent ?"

«Pourquoi croiriez-vous une femme que vous avez cessé de croire? et si je suis coupable, me coûterait-il d'ajouter un mensonge aux autres?» (p. 151) 
Devant ces esquives répétées, comment réagit Artus? Va-t-il céder? - Eh bien! non. Tout au contraire, il imagine une mise en scène rocambolesque: que Guenièvre donne à Lancelot un rendez-vous galant dans sa chambre! on verra bien... Convaincue que Lancelot, parti au Château noir, n'en pourra pas prendre connaissance, elle y consent (p. 153).

Hélas! il revient, découvre la convocation amoureuse. Il s'y rend. Artus, pour l'attendre, s'est couché dans le lit de la reine. Lancelot arrive. Il s'y couche aussi. Mais, au lieu des caresses de son amie et de l'amour, il rencontre les poings du mari et la mort...

L'amant et le mari, couchés ensemble: la situation frise le vaudeville, certes. Mais ne peut-on y voir aussi un nouvel écho du penchant bien connu de Cocteau?

Le vaudeville cependant toume bientôt au drame. Car Artus, furieux, porte à Lancelot de tels coups que « le meilleur chevalier » succombe (p. 160).

\section{Retour au couple $n^{\circ} 3$ : Lancelot et Guenièvre ou Mort et Immortalité}

Tout autre, on le sait, est la fin de Lancelot selon la Mort le Roi Artus. Là, le héros amoureux survivait à son roi, tombé au combat, emporté par les fées en l'île d'Avalon. Gauvain et Lionel, Mordret et ses fils, et tous les compagnons de la Table Ronde, succombent décimés. Seuls Gifflet et Lucain échappent à l'hécatombe. C'est le rouge crépuscule des temps chevaleresques. Et, tandis que la veuve Guenièvre se fait nonne - elle a passé soixante-dix ans, il est vrai -, Lancelot devient prêtre dans un ermitage. Il mourra pieusement et sera inhumé au Château de la Joyeuse Garde. - Fins édifiantes, s'il en est!

Nul pareil repentir ne visite les amants de Cocteau. Au contraire! Car les amants célèbres ne meurent jamais tout à fait : les couples fabuleux survivent immortels et s'unissent bientôt en des noces mystiques. Lancelot et Guenièvre se rejoignent, vivants ; mais dans cet au-delà du domaine du Lac.

Grande et belle scène poétique, quand le couple fameux descend de compagnie sous le voile des eaux dans ce royaume-fée! Car, estime la Reine, « il existe des mariages plus légitimes que ceux de la terre» (p. 162). Elle se couche contre le corps de Lancelot, apparemment défunt :

"Au fond du lac, dit-elle, je vois le chevalier que l'eau déforme. A travers les reflets et les moires on dirait qu'il bouge. Mon corps nouveau flotte à la surface. Il enfonce doucement. Et voici que Lancelot semble monter à ma rencontre. Je traverse des couches lumineuses. Des ombres m'escortent et m'empêchent de descendre vite. (...) Si vous saviez combien ce lac m'aspire avec douceur! (...) Il monte... Il monte... Il se précise. Ses yeux ouverts me regardent. Ses bras flottent et me saluent comme les plantes qui entourent sa couche. Mes oreilles deviennent sourdes. Mes jambes se posent sur ses jambes. Adieu, cher Artus. Lancelot! Me voilà » (acte III, p. 163)

Où, de nouveau, dans la tradition légendaire, Cocteau a-t-il pu glaner les prémices de cette belle vision? Peut-être a-t-il transposé ici un épisode recueilli dans les transpositions en français modeme des Romans de la Table Ronde. On y voit Lancelot sauter dans l'onde transparente d'un étang pour en sauver un chevalier tout armé et la dame qu'il tenait embrassée. La demoiselle qui l'accompagnait lui raconte alors que ces amants s'entr'aimaient fort. Mais le mari jaloux occit le chevalier rival et le jeta dans cet étang. Quand l'épouse l'apprit, elle implora son Dieu qu'il daignât lui montrer le corps de son ami. Celui-ci, alors, lui apparut ; et elle plongea le rejoindre dans l'eau (op. cit., tome II, XIVII, p. 152). - On discerne 
sans peine quelles similitudes rapprochent les deux épisodes: le mari jaloux, assassin; le chevalier noyé; l'amante qui le rejoint ; leur enlacement dans le royaume des eaux...

\section{Les Avatars du Mythe}

Précieuse, cette pièce de Cocteau! elle éclaire quelques lois qui gouvernent l'évolution des mythes.

Quels archétypes légendaires nourrissent les personnages qui animent sa version des Chevaliers de la Table Ronde? Cocteau sans doute n'a point puisé aux sources médiévales originales: on le voit mal déchiffrant le français du Moyen Age, encore moins le vieil hautallemand. Mais il a pu connaitre le Parzival de Wolfram à travers la traduction française qu'Emest Tonnelat en avait donnée chez Aubier-Montaigne. Sans doute est-ce à lui qu'il emprunte Ségramor, du moins son nom, sinon son rôle.

S'il a pu accéder aussi à maints épisodes du Lancelot-Graal, ce n'est certes point dans la monumentale édition d'Oskar Sommer, mais plutôt à travers les adaptations modemes, telle celle que Jacques Boulenger en avait proposée chez Plon. C'est là qu'il a pu trouver la Guenièvre bis, les amants sous l'eau, la figure de Merlin, etc. - Telles paraissent les deux résurgences principales où il a puisé pour composer son élixir personnel.

De ces données légendaires qu'a-t-il gardé ? Qu'a-t-il modifié ? Pour qu'un Mythe originel demeure reconnaissable, ses échos modernes doivent en conserver assez d'élémentsdes personnages, des épisodes, des intrigues - pour pouvoir l'identifier. Tel est le cas dans les Chevaliers de la Table Ronde de Cocteau.

Ainsi respecte-t-il quelques thèmes des plus fondamentaux de la vulgate légendaire: l'éducation de Lancelot par la Dame du Lac; le mariage d'Artus et de Guenièvre; le double coup de foudre de Guenièvre et de Lancelot; leur longue liaison secrète; l'intervention d'une fausse Guenièvre; la révélation à Artus de son infortune; la quête du Graal par Galaad, fils de Lancelot; sans oublier le Graal lui-même, qui voit se confirmer ses caractères constants, ses traits irréductibles: son introuvable tour, son éclat, son parfum, ses questions fatidiques, sa vertu révélatrice du Vrai, son dépassement des oppositions.

Mais autour de ces étoiles fixes, que de comètes errantes gravitent, captées par son attraction! Que de broderies inédites peut se permettre un auteur sur la trame traditionnelle! Cocteau ne s'en prive guère...

Divorce d'abord entre thèmes séculairement liés et leur concubinage scandaleux avec d'autres jusque là étrangers (le Coup douloureux dissocié du Gaste Pays, mais associé à l'épreuve du Siège périlleux). Dédoublements et substitutions de personnages ou d'objets (deux Guenièvre, deux Gauvain, deux Galaad, deux Graals, les vrais et les faux). Transfert de personnages d'un cycle romanesque ou d'une parentèle légendaire à l'autre (Lancelot devenu vrai fils de la Dame du Lac; Mélusine enlevée aux Lusignans; Sagremor importé de la dynastie byzantine pour l'incorporer au lignage d'Artus). Echanges et transpositions d'aventures (la Guenièvre-bis visant à troubler Lancelot plutôt qu'à séduire Artus; la noyade des amants attribuée à Guenièvre et à Lancelot même, devenu de spectateur acteur). Invention de personnages inconnus (Blandine); escamotage en revanche de figures connues (Mordred, Hélaine, le Roi Pêcheur). Promotions inédites à la Table Ronde (Beaudémagu, Ségramor, Merlin). Renversement du caractère de talismans (le Graal réputé maléfique) ou 
de héros essentiels (Merlin transformé de protecteur du royaume d'Artus en agent de sa dévastation).

On voit quelles « libertés grandes » s'arroge vis-à-vis de la tradition légendaire le cocktail concocté par Cocteau. Variations tantôt heureuses, tantôt plus discutables. II n'est pas sûr que tous reconnaissent aisément le Mythe fondamental sous ces oripeaux insolites. Mais c'est la suprême désinvolture des Grands.

Travaillant par réminiscences, Cocteau prit ensuite conscience des distorsions qu'à son insu il avait infligées au Mythe, et il en assuma la responsabilité. I a raconté cette élaboration:

« En 1934, j'étais malade (...) Un matin, (...), j'assistai d'un bout à l'autre à ce drame dont l'intrigue, l'époque et les personnages m'étaient aussi peu familiers que possible. (...). Une fois la pièce écrite, je me documentai, je me trouvai en face de mes fautes de fabuliste et je décidai de m'y tenir." (Préface, p. 72)

C'est excellement dit. Et pensé.

Pourquoi un tel écart entre le scénario canonique et la version moderne ? C'est que chaque siècle a ses mours; chaque auteur, ses goûts.

Les temps, d'abord, changent, ont changé. Révolu, l'amour courtois d'un chevalier sigisbée dont les attentions pour sa Dame flattent la vanité de l'époux. La mentalité bourgeoise s'accommode à l'occasion de l'« adultère mondain ", surtout s'il se prévaut d'une passion romantique que sanctifie une fidèle liaison. Ainsi Cocteau tend à rendre moins choquantes aux yeux de son époque certaines données de l'intrigue.

Ainsi gomme-t-il certains aspects déplaisants des relations de Lancelot avec l'épouse de son roi. I tait l'aventure incestueuse d'Artus avec Morgane. Il supprime l'amour initial de Guenièvre pour Artus. Il efface l'infidélité involontaire de Lancelot avec la porteuse du Graal. Ainsi parachève-t-il la «moralisation » par l'amour unique, entamée déjà par les conteurs médiévaux. Sans doute ce mariage de convenances entre prince et princesse, sans amour, avec accord préalable de tolérance mutuelle, cette idéalisation romantique de la passion pour le seul ami de cour, cela sent-il un peu l'hypocrite morale bourgeoise. Et il est un brin surprenant de trouver pareils scrupules chez Cocteau.

De même pour la naissance de Galaad: le "Très Pur " n'est plus fruit illégitime d'une passade nocturne entre un Lancelot amant de la reine et une jeune vierge. Il devient fils légitime de noces régulières de Lancelot avec une Fée, noces conclues et rompues avant la rencontre foudroyante avec Guenièvre.

Cette moralisation ou normalisation de l'intrigue est-elle toujours réussie? Ce n'est point si sûr. Le succès n'en parait que relatif, tant il est difficile de jamais toucher au Mythe où tout se tient, sans que s'en répercutent ailleurs d'imprévisibles disgrâces. - Un Mythe se défend!

Souvent aussi le siècle se manifeste allergique au merveilleux. Ainsi est éliminé le philtre magique qui persuade Lancelot qu'il étreint la reine, alors que c'est Hélaine qu'il tient dans ses bras. Sans doute celle-ci, simple mortelle, n'est guère qu'un nom dans la pièce. Fée qui ne bénéficie d'aucun privilège particulier.

Se vérifie aussi par là une loi des survivances mythiques : tout se passe comme si tout effort pour évacuer du Mythe ses éléments surnaturels et pour le plier au carcan réaliste sur tel ou tel point, était quasi automatiquement compensé par une résurgence d'éléments archai- 
ques sur tel ou tel autre point. Le Mythe résiste. Il se venge de qui veut l'asservir à un monde qui n'est pas le sien. Le Perlesvaus n'en est pas non plus un mauvais exemple.

Souvent aussi un auteur incline le Mythe dans le sens de ses tendances particulières. Minces, parfois, les limites entre innovations heureuses et disgracieuses distorsions. Il faut savoir transformer sans déformer, transfigurer sans céder au difforme. Si un Gracq historien veut réviser l'aventure du Graal pour la mieux plier aux lois de l'Histoire humaine, un Cocteau aussi y a imprimé l'impact de ses penchants personnels. Lui qui, opiomane, dut se libérer de l'emprise des stupéfiants, il assimile la quête du Graal à une cure de désintoxication.

Quant au démon mâle Ginifer qui, métamorphosé en fausse Guenièvre, prétend à l'amour de Lancelot, quant à la scène du mari «cocu » couché avec l'amant de sa femme, ces curieuses inventions symbolisent peut-être les phantasmes particuliers de l'auteur. Sans oublier que c'était Jean Marais, l'« ami » de Cocteau, qui incarnait le « Très Pur »...

Trop annexer la légende à des soucis trop personnels convainc mal. Rien, ici, dans le Mythe originel, n'autorisait si gratuites déviations. Un Mythe résiste. Surtout quand on veut l'accaparer à des fins qui lui sont par trop étrangères.

Maints avatars de la légende procéderaient enfin du goût de Cocteau pour l'illusionnisme. La critique s'étonna de tant de dédoublements et substitutions: une fausse Guenièvre, mais aussi un faux Gauvain, un faux Galaad, et, pour comble, un faux Graal! Labyrinthe de reflets et de miroirs, où on doit reconnaître qu'on s'égare un peu...

Mais est-ce là pur illusionnisme, gratuit? Et si ce jeu d'apparences trompeuses reflétait une philosophie profonde de l'auteur? Si tout le monde sensible n'était, justement, qu'illusions, voile de la « maya »? Et s'il nous fallait trouver un Graal, c'est-à-dire la Poésie, pour nous démasquer la vraie vie, la vraie réalité ? - Telle est la tâche, révélatrice, du Poète. Telle fut l'Esthétique constante, la Poétique de Cocteau :

" (La poésie) nous dévoile dans toute la force du terme. Elle nous montre nues, sous une lumière qui secoue la torpeur, les choses surprenantes qui nous environnent et que nos sens enregistraient machinalement. "s

Attitude quasi bergsonienne, qui se rencontre dès 1926 dans le Rappel à l'ordre ${ }^{19}$.

Enfin, se confirme une loi du genre, déjà dégagée ailleurs ${ }^{20}$. Dans une constellation légendaire, comptent non point tant les rapports consacrés entre les thèmes que la présence de nombre d'entre eux, d'une façon ou d'une autre, au sein de cette constellation, - quitte à ce qu'entre ces motifs permanents se créent des rapports nouveaux. Ainsi, le Coup douloureux, d'habitude associé au «Gaste Pays », s'en trouve ici dissocié. Et le voilà par contre associé au thème, initialement tout étranger, du Siège périlleux. Suffit que ces trois motifs: Gaste Pays, Coup douloureux, Siège périlleux, y paraissent (De même, que de figures diverses les conteurs médiévaux prêtent à la constellation des Talismans merveilleux! Lance flamboyante, Graal éblouissant, Epée brisée (ou leurs équivalents) sont généralement présents, mais selon de variables rapports. Suffit qu'ils y soient!).

\footnotetext{
${ }^{19}$ Voir le Secret professionnel, le Discours de réception à l'Académie francaise, le Discours d'Oxford, le Rappel à l'Ordre, la Difficulté d'être, Joumal d'un inconnu, etc.

${ }^{20}$ Voir notre étude : Robert Bodri (sic), « Originalnost pripovedaca u odnosu na vecite teme cudesnog », Knjizevna Kritika, 1981, n 1, janv-févr., pp. 5-18.
} 
C'est au prix de tels renouvellements, on le sait aujourd'hui, qu'un Mythe survit dans l'imagination huamine. Notre vie physiologique aussi ne se maintient que par le renouvellement complet de nos cellules. A cela se mesure la plasticite éternelle des Mythes: s'ils restent assez souples pour s'adapter, sans changer leur nature, à de multiples avatars. De là naît le charme profond qui séduit en eux les imaginations humaines. C'est qu'ils éveillent tout à la fois et l'attente et la surprise. Attente de l'auditeur, comblé de retrouver les vieux thèmes ancestraux qui ébranlent sa sensibilité avec une infaillible efficacité. Mais surprise en même temps de les voir transcendés par d'inédites triangulations. Double et contradictoire action, par où le mythe exerce son impact toujours si fatal sur nos coeurs, en en exaltant la puissance comme à la factorielle de son nombre.

\section{Leçons}

Ainsi l'œuvre moderne véhicule sur le Mythe d'instructives leçons.

Pour tous, médiévistes ou modernistes, ces reprises contemporaines de légendes anciennes jouent un rôle précieux de révélateur. Pour les médiévistes, elles dégagent l'essence permanente, le noyau irréductible des mythes, celui auquel ne parvient à attenter nulle tentative de rénovation. Pour les dix-neuviémistes ou vingtiémistes, elles trahissent mieux que tout, par les distorsions qu'il leur inflige, les obsessions personnelles de l'auteur d'aujourd'hui.

Bref, le Mythe, tantôt, sur certains points, résiste. Tantôt, sur d'autres, il s'accommode de modifications, pourvu toutefois qu'elles demeurent heureuses, orientées dans un sens parallèle à la pensée mythique. Mais veut-on le gauchir, prétend-on le démythifier, et s'il còde à ces visées rationalisantes ou réalisantes, une compensation quasi automatique le fait se revenger ailleurs, sur d'autres points, réinvestissant par de nouveaux éléments mystiques la version ainsi « aplatie ».

Doué d'une vie propre, le Mythe évolue.

Mais il reste LE Mythe. 